\title{
A Proportional Hazard model to establish factors that are significant in child survival
}

\author{
Musizvingoza Ronald ${ }^{\dagger 1}$, Mwembe Desmond ${ }^{\dagger 2}$, Nyamugure Philimon ${ }^{\dagger 3}$ \\ ${ }^{1,2,3 *}$ Department of Statistics and Operations Research, National University of Science and Technology. \\ P O Box AC 939 Ascot, Bulawayo, Zimbabwe
}

\begin{abstract}
This study addresses important issues affecting under-five mortality in Zimbabwe. The objective of this research is to establish factors that are significantly impacting on child survival and to determine the survival rate of children under the age of five years. Cox regression and Kaplan-Meier estimator were used for data analysis. Child survival was significantly influenced by two predictor variables, breastfeeding and immunisation status $(p<0.05)$. The Hazard ratios for variable breastfeeding and immunisation are 2.806 and 4.778 respectively. The survival functions for the children indicate a high survival rate especially in children who are well breastfed and those who are fully immunised. This study supports health policy interventions that enhance child survival. Immunisation and breastfeeding should be encouraged among mothers to enhance child survival.
\end{abstract}

Keywords: child survival, Cox Regression, factors, , Kaplan-Meier, hazard ratio

\section{Introduction}

Child Survival remains a principal objective for every country especially developing countries. In Zimbabwe, under-five mortality rates decreased substantially after Independence in 1980, but began to increase in 1996, possibly in response to the start of economic challenges and the introduction of cost-recovery policies (Ministry of Social Services 2010). ZIMSTATS (2009) estimates under-five mortality rate of 94 deaths per 1000 live births. Children are the most vulnerable members of the society. About 80 percent of deaths among underfive children in Zimbabwe are caused by four mostly preventable causes: Neonatal causes (29\%); AIDS (21\%); pneumonia (13\%), diarrhoea (9\%) and Measles 8\%. (Ministry of Social Services 2010). Young children are sensitive not only to their environment but they can be affected by the health and well being of their mothers and also the quality of health services and support offered to them. Under-five mortality is certainly one of the most expressive indices of development of a country UNICEF (2005)

\subsection{Background Of The Study}

Decline in under-five deaths in Africa has been slow, despite various approaches to improving child health WHO (2005). In past decades, many countries adopted strategies aimed at preventing under-five mortality. Zimbabwe also adopted these strategies under its policy paper, Child survival strategy 2010-2015. As part of the millennium development goals for health, nations pledged to ensure a two third reduction in underfive mortality by 2015 from the levels of 1990. For Zimbabwe this translates to a reduction from 79 to 27 deaths per 1,000 live births in 1990 to 2015 respectively. Due to the worsening of the socioeconomic conditions of most countries especially in the sub-Saharan Africa the result has been high levels of under-five mortality and there still remains a big challenge in combating it. Over the years, the Ministry of Health and Child Welfare has adapted and adopted a number of high impact and low cost strategies aimed at reducing the unacceptably high rates of childhood deaths. One such intervention is the Integrated Management of Childhood and Neonatal Illnesses (IMCNI), adopted and institutionalized in 1999 to address child health problems and ensure maximum development of the child. The overall objective of the strategy is to contribute towards the reduction of child mortality. IMCNI, as an integrated approach to child health, combines improved management of common childhood illnesses with aspects of nutrition, immunization and other important factors influencing child health, including maternal health. It has three components, covering health worker skills development, health systems performance improvement and a third component involving the household and community in child survival issues. The implementation of the IMCNI strategy followed the WHO guidelines which included an introductory phase, early implantation phase and expansion phase. Implementing the strategy required collaboration between programmes and between different levels of the health system.

Zimbabwe is faced with a high rate of child deaths and children are dying with known causes. There is a great misunderstanding in the different factors affecting child survival. Although several intervention strategies have been adopted to curb child deaths we still have high child mortality.

This paper aims at carrying out a survival analysis of children under the age of five years in Zimbabwe 
The objectives of the research are:

1. To establish factors that are significant in Child survival.

2. To estimate the survival function of children under the age of five years

3. To estimate survival patterns of under five mortality by each significant factor

Mosley and Chen (1984) proposed a two level analytical framework. The framework organizes factors associated with infant mortality into either proximate or distal determinant categories. The proximate level includes biomedical and biodemographic factors which are directly associated with infant and child deaths. Proximate determinants typically involve the interaction of malnutrition and infectious diseases and adverse reproductive characteristics of mothers (maternal factors). The distal level includes social, cultural and economic conditions, such as the association between infant mortality and maternal education and family occupation, which have been found to affect child survival in many developing countries. The Mosley-Chen framework has served as a useful starting point for theory, research and policy making over the last decade

Klaauw and Wang (2004) developed a flexible parametric framework for analyzing infant and child mortality. This framework is based on widely used hazard rate models, which they extend with two features. First, the model allows individual characteristics and household's socio-economic and environmental characteristics to have different impacts on infant and child mortality at different ages. Second, they allow for frailty at multiple levels, which can be correlated with each other. The first feature seems to be particularly relevant in describing infant and child mortality, child specific. They also use the estimated model to perform a number of policy experiments. The key findings were that:

1. The use of unclean cooking fuels (wood and coal) significantly reduces the neonatal survival probability in rural areas.

2. Access to safe water or sanitation reduces child mortality risks by about 34 per cent in rural areas.

3. A higher maternal education level reduces child mortality and female education has strong health externalities.

4. Access to safe water and sanitation, and immunization reduce diarrhoea incidence in rural areas, while access to modern sanitation facilities (flush toilets) reduces diarrhoea prevalence in rural areas.

5. Significant linkages between Acute Respiratory Infections (ARI) incidence and use of unclean cooking fuels are found using the city level data constructed from the survey.

Rutstein (2008) using multivariate analyses examined the association between birth intervals and infant and child mortality and nutritional status. He used both bivariate and multivariate designs to Demography and Health Survey data from Bolivia, Guatemala, and Peru. The results suggested that children born after birth intervals of 24-29 months still face under-five mortality risks that are 70-90\% higher than children born after intervals of 36-41 months.

Mutunga (2007) explored the relationship between household's environmental and socio-economic characteristics on child mortality. He argues that infant and child mortality is related to the household's environmental and socio-economic characteristics, such as mother's education, source of drinking water, sanitation facility, type of cooking fuels and access to electricity. He used a Weibull hazard rate framework to analyze the determinants of child mortality and he came up with the following model. The hazard function of the Weibull model was defined as follows:

$$
h(\mathrm{t})=\alpha \lambda t^{\alpha-1}
$$

Where $\lambda=\exp \beta^{\prime}(X) . \alpha$ is a scale parameter $\lambda<1$, indicates that the hazard falls continuously over time, while $\lambda>1$ indicates the opposite. The results of the paper showed that a household's environmental and socio-economic characteristic are found to have a significant impact on child mortality. He recommended that policies aimed at achieving the goal of reduced child mortality should be directed on improving the household's environmental and or socio-economic status if this goal is to be realized.

Bawah, A.A. etal (2009) used time conditional Weibull models to conclude that immunisation reduces child mortality. Their model estimated parameters of the proportional hazards model, assuming that the hazard rate for childhood mortality is the product of a baseline failure rate, $h_{0}(t)$ which is a function of time only, and a positive function $g(x, A)$, independent of time, which incorporates the effects of a vector $X$ comprised of covariates, such as immunization vaccine, mother's characteristics (age and educational attainment), and an index of poverty as estimated by the method of principal components. The conditional hazard is given by:

$$
h(t / \underline{X})=h_{0}(t) \cdot g(\underline{X}, \underline{A})
$$

Where $\underline{X}$ is a row vector consisting of the covariates 


$$
\underline{X}=\left(x_{1}, x_{2} \ldots, x_{m}\right)
$$

$\underline{A}$ is a column vector consisting of the unknown parameters of the model compounded over $T$ units of time.

$$
\underline{A}=\left(a_{1}, a_{2} \ldots, a_{m}\right)^{T}
$$

And where $m=$ the number of covariates that are time-independent. Effects of immunization accumulated with the incremental provision of vaccines as infancy progresses. Corresponding effects were posited to accumulate with age $t$, starting with the baseline failure rate given by the Weibull underlying hazard, with "shape parameter" $\beta$

$$
\lambda_{0}=\frac{\beta}{\eta}\left(\frac{\mathrm{t}}{\eta}\right)^{\beta}-1
$$

and the conditional hazard given by:

$$
\lambda(t, X)=\beta t^{\beta-1} e^{\sum_{j=0}^{m} a_{j} \cdot x_{j}}
$$

Bawah etal. concluded that all vaccines exhibit large and statistically significant effects on child mortality. They also noted that the risk of dying for children younger than five is reduced by more than $70 \%$ among those who are fully immunized compared with those who are not. Partial immunization also prevents mortality. The implications of the pronounced immunization effect on cumulative survival was further explained by a Kaplan-Meier cumulative survival curve depicting the main effects of childhood survival by immunization status (full, partial, or none), with statistical adjustment for maternal age and educational attainment and relative household economic status. The Kaplan-Meier estimates showed that children who have received all vaccinations experience substantially higher cumulative survival odds than their counterparts who are not fully immunized. Those who have received no vaccinations experience catastrophically high cumulative risks of dying by the time they reach age five. Partial immunization reduces cumulative risk to $20 \%$, and full immunization leads to a further reduction of cumulative mortality risk to about $10 \%$.

Worku (2009) used Pearson's Chi-square tests, survey logistic regression analysis and Cox proportional hazards model to analyse the significant impacts of the factors affecting under-five mortality in South Africa. The study identified key predictors of mortality among children under the age of five years. The results of the paper showed that under-five mortality was significantly influenced by three predictor variables (duration of breastfeeding, marital status, and ownership of flush toilet

Kembo and Ginneken (2009) used multivariate proportional hazard analysis to analyse the impact of maternal, socioeconomic and sanitation variables on infant and child mortality in Zimbabwe. Their study addressed important issues in infant and child mortality in Zimbabwe. Their results showed that births of order $6+$ with a short preceding interval had the highest risk of infant mortality. The infant mortality risk associated with multiple births was 2.08 times higher relative to singleton births $p \leq 0.001$ Socio-economic variables did not have a distinct impact on infant mortality. Determinants of child mortality were different in relative importance from those of infant mortality. They recommended health policy initiatives that stimulate the use of family planning methods to increase birth spacing. However the study did not fully explore all the factors and determining their significant impact on child survival.

\section{Materials And Methods}

The study uses Zimbabwean live births who:

- Were born five years preceding the 2005/06 Zimbabwe Demography and Health Survey

- Either survived up to the age of five years or not.

\subsubsection{Model Assumptions}

1. The hazard ration is the same all the way throughout the study

2. Any factor which affects death of a child does so by the same ratio all the times.

\subsubsection{Empirical Model}

Cox Proportional Hazard model was used in this study to establish factors that affect child survival among the set of selected bio demographic variables and also to estimate the survival function of children under the age of five years. Proportional hazard models allow us to simultaneously test the effect of several variables on child survival. The Kaplan-Meier estimator was used to estimate the survival function of children in different groups under the age of five years. 


\subsubsection{Explanatory Variables.}

Following the analytical framework developed by Mosley and Chen (1984), the literature on infant and child mortality has established a set of individual and socio-economic factors that have shown to influence the probability of early childhood death. This study uses variables available in the 2005-2006 Zimbabwe Demography and Health Survey. The bio demographic variables used are sex of the child, birth weight, age of mother at birth, breastfeeding status and immunisation status of the child.

The explanatory variables were categorized before starting the analysis. Sex of the child was categorised as either male or female. In the data collection, birth size was categorised into very small, small, average size, large and very large size. However for the purpose of analysis in this study birth weight was classified as less than or more than $2500 \mathrm{~g}$. Immunisation status is classified as fully immunised or not fully immunised. To be fully immunised a child should have received one dose of BCG vaccine, three doses each of DPT and polio vaccines and one dose of measles vaccine. Age of mother at birth was categorised into four groups: less than 20 years, 20-29 years, 30-39 years and 40-49 years. These breakpoints were selected because of the need to cover the different reproductive groups: adolescents, young adults, adults and old adults. Breastfeeding status was classified as never breastfed and breastfed.

Table 1: Explanatory Variables and their Coding

\begin{tabular}{|l|l|}
\hline Biodemographic Variable & Definition and Coding \\
\hline Sex of the Child & Male $(1)$, Female $(0)$ \\
\hline Birth Weight & $<2500 \mathrm{~g}(0),>=2500 \mathrm{~g}(1)$ \\
\hline Breastfeeding Status & Ever $(1)$, Never $(2)$ \\
\hline Maternal Age & $<20 y r s(0), 20-29 y r s(1), 30-39 y r s(2), 40-40 y r s(3)$ \\
\hline Immunisation Status & Fully(1) Not Fully (0) \\
\hline
\end{tabular}

The dependant variable is the hazard ratio (relative risk) of dying for a child before reaching the age of five years.

\subsubsection{Model Specification}

The study will use the proportional hazard model to establish factors that are significant in child survival among children under the age of five years in Zimbabwe. The factors can be modelled by the following multiple regression model.

$$
Y=\beta_{0}+\beta_{i 1}+\beta_{2} X_{i 2}+\beta_{3} X_{i 3}+\beta_{4} X_{i 4}+\beta_{5} X_{i 5}
$$

Where $Y=$ the dependent variable, is the relative risk of dying for a child before they reach the age of five years. $\beta_{0}, \beta_{i}, \beta_{2}, \beta_{3}, \beta_{4}, \beta_{5}$ represents the proportional change in the risk of death of a child due to the changes in the explanatory variables. The characteristics for each child in our model or the explanatory variables will be represented by $X_{i} . X_{1}$ represents sex of the child such that:

$$
X_{1}= \begin{cases}0 & \text { Male } \\ 1 & \text { Female }\end{cases}
$$

$X_{2}$ represents age of mother at birth of the child and it is categorised as follows

$$
X_{2}=\left\{\begin{array}{lc}
0 & <20 \text { years } \\
1 & 20-29 \text { years } \\
2 & 30-39 \text { years } \\
3 & 40-49 \text { years }
\end{array}\right.
$$

$X_{3}$ represents birth weight of the child as follows:

$$
X_{3}=\left\{\begin{array}{l}
0 \quad<2500 g \\
1 \quad \geq 2500 g
\end{array}\right.
$$

$X_{4}$ represents breastfeeding status of the child and will be categorised as follows:

$$
X_{4}= \begin{cases}0 & \text { Not Breastfed } \\ 1 & \text { Breastfed }\end{cases}
$$

$X_{5}$ represent the immunisation status of a child and will be categorised as below: 


$$
X_{5}= \begin{cases}0 & \text { Not Fully Immunised } \\ 1 & \text { Fully Immunised }\end{cases}
$$

The study assumed the model fitted has a proportional hazard relationship so the proportional hazard model for the children with above mentioned predictors becomes:

$$
h(t)=h_{0} \exp \left(\beta_{0}+\beta_{1} X_{1}+\beta_{2} X_{2}+\beta_{3} X_{3}+\beta_{4} X_{4}+\beta_{5} X_{5}\right)
$$

$h(t)$ is the hazard or risk of a death for a child, with the above set of explanatory variables and is dependent on (or determined by) the five explanatory variables or covariates $\left(X_{1}, X_{2}, X_{3}, X_{4}, X_{5}\right.$ ) whose impact on the survival of the child until the age of five years is measured by the size of the respective coefficients $\beta_{0}, \beta_{i}$, $\beta_{2}, \beta_{3}, \beta_{4} \beta_{5}$. The baseline hazard at time $t, h_{0}(t)$ is the value of the hazard if all the explanatory variables are equal to zero (the quantity $\exp (0)=1$ ).

By dividing both sides of the above equation by $h_{0}(t)$ and taking logarithms, we obtain:

$$
\log \left(\frac{h(t)}{h_{0}(t)}\right)=\beta_{0}+\beta_{1} X_{1}+\beta_{2} X_{2}+\beta_{3} X_{3}+\beta_{4} X_{4}+\beta_{5} X_{5}
$$

The fraction $\frac{h(t)}{h_{0}(t)}$ is the hazard ratio which is the risk of death of a child.

The ratio $\frac{h(t)}{h_{0}(t)}$ measures the risk of death for a child at time $t$ before reaching the age of five years. The key assumption of proportionality in the model implies that the quantities $\exp \left(\beta_{i}\right)$ are the hazard ratios. The study interprets this hazard ratio as the sudden death of a child who has survived until some time $t$. For investigating whether the selected factor is significant, we test the null hypothesis:

$$
H_{0}:\left(\beta_{1}, \beta_{2}, \beta_{3}, \beta_{4}, \beta_{5}\right)=0
$$

Against the alternative hypothesis:

$$
H_{1}:\left(\beta_{1}, \beta_{2}, \beta_{3}, \beta_{4,} \beta_{5}\right) \neq 0
$$

\subsubsection{Kaplan-Mier Estimator}

For estimation of the survival function, $S(t)$ of children under the age of five years by each selected factor we use the Kaplan-Meier estimator also called the product limit estimator. The time period in this study is from 0 months to 60 months. The survival times, including censored observations, of a homogeneous group of children are $t_{1}, t_{2}, \ldots, t_{k}$ for $k=1,2,3 \ldots 60$ months represents the survival times of children. The survival times should be ordered such that:

$$
t_{1}<t_{2}<t_{3}<\ldots<t_{k}
$$

For a given value $t$ there is a large value $T$ which is the maximum number of months under study that is 60 months such that $t<T$. As events are assumed to occur independently of one another, the probabilities of surviving from one interval to the next for a child may be multiplied together to give the cumulative survival probability, the probability $\hat{S}(t)$ is estimated as follows:

$$
\hat{S}(t)=\frac{r_{1}-d_{1}}{r_{1}} \times \frac{r_{2}-d_{2}}{r_{2}} \times \ldots \times \frac{r_{k}-d_{k}}{r_{k}}
$$

where: $r_{k}$ is the number of children alive just before time $t_{k}$ (the $k^{\text {th }}$ ordered survival time).

$d_{k}$ denotes the number of children who died at time $t_{k}$

\section{Data Analysis And Results}


Summary statistics based on the 2005/06 ZDHS data allow us to understand under-five mortality rates according to the factors that are included in our analysis. While this does not tell us anything about causalities or the exact magnitude of a correlation and its statistical significance, it provides a first overview of the incidence of under-five mortality in different contexts. The total live births that occurred in the ten year period preceding the survey was 9491. In this research the sample is limited to 5077 live births that occurred in the five years preceding the survey. This translates to $53 \%$ of total live births that occurred. The 5 year period was considered mostly because data was missing on other indicators that define under-five mortality.

Total live births were 5077 as shown in table 2, sex was evenly distributed, male constituted $51 \%$. The majority of the children born during this period were of normal birth weight $(88 \%)$. Most births were attributed to mothers between 20-29 years, (58\%), however teenage births contributed $7 \%$ to total live births. Breastfeeding among mothers was high at $98 \%$. Immunisation among the children was relatively high because $79 \%$ of the children were fully immunised.

Table 2: Distribution of Biodemographic Variables.

\begin{tabular}{|l|l|l|}
\hline Biodemographic Covariate & Number of Live Births & Percentage Distribution \\
\hline Sex of Child & & 50.7 \\
Male & 2572 & 49.3 \\
Female & 2404 & \\
\hline Birthweight & 626 & 12.3 \\
$<2500 \mathrm{~g}$ & 2404 & 49.3 \\
$\geq 2500 \mathrm{~g}$ & & \\
\hline Maternal Age & & 7.0 \\
$<20$ years & 358 & 58.4 \\
20-29 years & 2965 & 28.2 \\
30-39 years & 1430 & 6.4 \\
$40-49$ years & 324 & \\
\hline Breastfeeding Status & & 98.1 \\
Breastfed & 4980 & 1.9 \\
Never Breastfed & 96 & 79.0 \\
\hline Immunisation Status & 4011 & 21.0 \\
Fully Immunised & 1066 & 100 \\
Not Fully immunised & 5077 & \\
\hline Total & & \\
\hline
\end{tabular}

Most children died before they reached their first birthday, Fig 1, infant mortality contributed $77 \%$ of the total number of deaths that occurred in the five year period

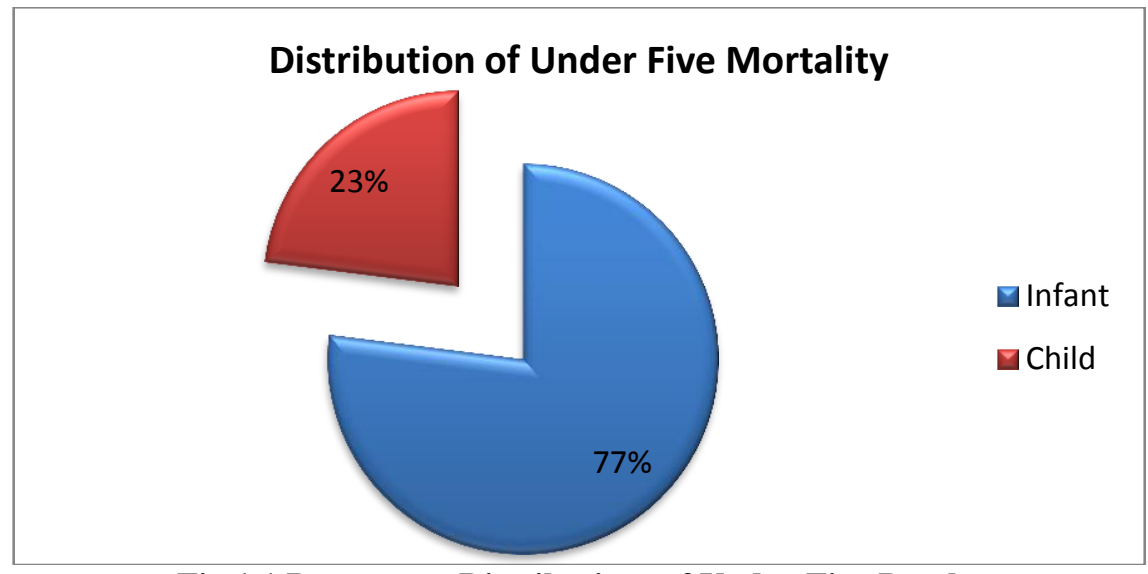

Fig 1.1 Percentage Distributions of Under-Five Deaths

3.1 Multivariate Proportional Hazard Analysis

Results from the Cox regression model Table 3, showed that only two factors breastfeeding and immunisation were statistically significant among all the factors. We found out that insufficient breastfeeding has a strong and highly statistically significant effect on increasing under five mortality $(\mathrm{p}=0.04$, CI 1.0167.749). The hazard ratios is 2.806 , which means that a mother who does not breastfeed her child is 2.805 times as likely to have lost a child as another woman who breastfeeds her child. The ratio also shows that breastfeeding a child will reduce the risk of dying by $35.6 \%$. Immunisation status of child exhibit statistically significant differences among fully immunised children and those who were not fully immunised ( $p=0.006 \mathrm{CI}$ 1.570-13771). A hazard ratio of 4.778 for the immunisation factor shows that a mother with a child who is not 
fully immunised is 4.778 times more likely to lose the child to death before reaching the age of five years as compared to a mother who fully immunise her child. This also indicates that by fully immunising a child a mother can reduce the risk of death in that child by $21 \%$. The results of the Cox model showed that the remaining factors sex of the child, birthweight and maternal age were not significantly affecting under five mortality with $\mathrm{p}$ values of $0.837,0.805$ and 2.804 respectively

Table 3: Estimates of Variables in the Cox Regression

\begin{tabular}{|c|c|c|c|c|c|}
\hline Covariate & B & SE & Sig & Exp(B) & $95 \%$ CI \\
\hline Sex & -0.107 & 0.517 & 0.837 & 0.899 & $0.327-2.474$ \\
Birthweight & 0.202 & 0.818 & 0.805 & 1.224 & $0.246-6.080$ \\
Maternal Age & & & 2.804 & 0.423 & \\
Maternal Age (1) & 0.983 & 1.299 & 0.449 & 2.672 & $0.210-7.448$ \\
Maternal Age (2) & 0.770 & 0.746 & 0.302 & 2.160 & $0.501-9.313$ \\
Maternal Age (3) & 0.914 & 0.565 & 0.106 & 2.495 & $0.824-7.556$ \\
Breastfeeding (3) & 1.032 & 0.518 & 0.047 & 2.806 & $1.016-7.749$ \\
Immunisation & 1.5642 & 0.539 & 0.004 & 4.778 & $1.661-13.738$ \\
\hline
\end{tabular}

For further analysis of the survival rate of the children we exclude all the other factors that are not significant and then we come up with a model of significant factors. The model for under- five mortality from the proportional hazard models will be of the form

$$
\log \left(\frac{h(t)}{h_{0}(t)}\right)=1.032 \text { Breastfeeding }+1.564 \operatorname{Im} \text { munisation }
$$

\section{Kaplan-Meier Estimator}

Children who have received all vaccinations experience substantially higher cumulative survival odds than their counterparts who are not fully immunized. Fig 2 shows that after 50 months about $90 \%$ of children who are fully immunised would still be alive but less than $50 \%$ of those who are not fully immunised would have died. The mean survival time of fully immunised children is about 56 months with a $95 \%$ Confidence Interval (52.692-58.767). Fully immunised children had a high survival rate of nearly 0.9, this means that immunised children had a $90 \%$ chance of surviving until the age of five years. For children who were not fully immunised the survival rate was low. The mean survival time for a child who is not fully immunised was 42 months with a 95\% Confidence Interval (34.649-50.648). The median survival rate of this group was 48 months with a confidence interval of 30.419-65.581.

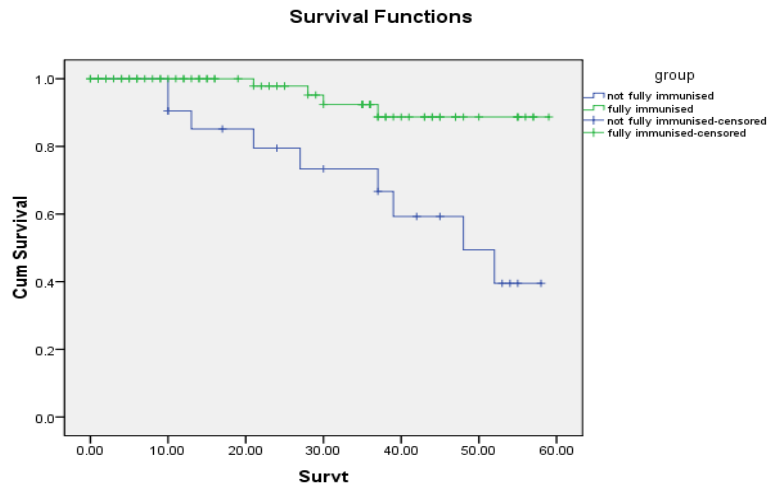

Fig 2: Cumulative Survivals by Immunisation Status

Cumulative survival rate for children who are breastfeed after a period of 3 years is about 0.9 as compared to those who are not breastfed who have a $70 \%$ chance of surviving past that time, Fig 3. The cumulative survival rate for a child who is breastfeed is 0.9 meaning they have a $90 \%$ chance of surviving until the age of five years. Children who were not breastfed had a survival rate of 0.6 


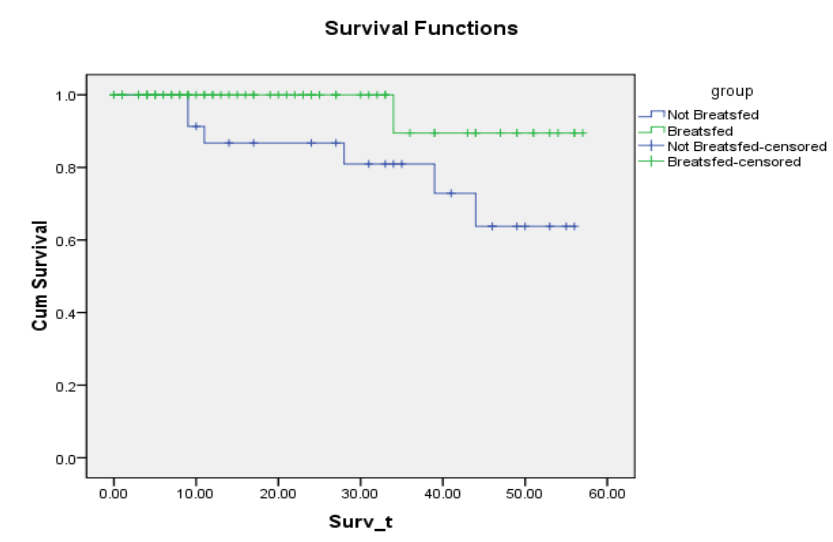

Fig 3: Cumulative Survivals by Breastfeeding Status.

\section{Discussion}

The study managed to establish factors that are significant in explaining child survival in Zimbabwe among the set of selected biodemographic variables. This study of survival rates of children in Zimbabwe revealed that negligence of immunization and breastfeeding affect survival rates of children. The role of child survival strategies like immunization and early initiation of breast feeding in improving survival cannot be challenged. The remaining factors sex, birthweight and maternal age were found to be insignificant at 5\% level of significance in causing under-five mortality. Children who are breastfed exhibit a higher chance of survival than their counterparts who are not breastfed. This research also found that in Zimbabwe absence, of immunisation is strongly negatively associated with child survival. Children who are not fully immunised have a low survival rate. The estimate of the survival functions at age 5 years was low and the hazards were high in children under the age of five years. The results showed that all the children have a 0.55 probability of surviving until the age of five years. The Kaplan-Meier estimator showed that immunisation and exclusive breastfeeding also enhanced the chances of surviving in children. The survival function for both fully immunised and breastfed children showed that most children in these groups have a high chance of surviving.

\subsubsection{Recommendations}

Overall progress of society and nations largely depends on declined trend of under-five mortality. Biodemographic characteristics such as breastfeeding status are the momentous factors that are keeping exceedingly high impact on child survival. Therefore, the following recommendations can be suggested based on the present study. It may help planners and policy makers to take appropriate decision to increase child survival in Zimbabwe.

1. Campaigns for breastfeeding should be held even for women or girls who are likely to have children in the near future. Exclusive breastfeeding should be compulsory for all women with children.

2. Universal immunisation coverage should be adopted for all children regardless of socioeconomic backgrounds. Children should receive all vaccines to enable them to fully protect themselves from the child killer diseases.

3. Stakeholders in field of child health should focus their energy and resources in designing strategies aimed at enhancing child survival. These may include access to free child, maternal and antenatal services.

\section{References}

[1]. Bawah, A.A., Phillips, J.F., Adjuik M., Vaughan-Smith, M., MacLeod, B., Binka. F.N. (2006) The Impact of Immunization on the association between poverty and Child Survival: Evidence from Kassena-Nankana District of Northen Ghana. Population Council Policy Research Paper 2006 No. 218

[2]. Government of Zimbabwe Ministry of Social Services, (2010) Millennium Development Goals Status Report for Zimbabwe 2010.

[3]. Kembo, J. and Van Ginneken, J.K. (2009). Determinants of infant and child mortality in Zimbabwe: Results of multivariate hazard analysis. Demographic Research Vol 21 Article 13 Pages 367-384

[4]. Klaauw, V.B and Wang, L. (2004) Child Mortality in Rural India (April 21, 2004).World Bank Policy Research Working Paper No. 3281 .

[5]. Kwabena, A. K. (2011) Socio-Economic Factors Affecting Under Five Mortality in South Africa-An Investigative Study. Journal of Emerging Trends in Economics and Management Sciences (JETEMS) 2 (2): 104-110

[6]. Mosley, W.H. and Chen, L.C. (1984) An analytical framework for the study of child survival in developing countries. Population and Development Review 10:25-45. doi:10.2307/2807954.

[7]. Mutunga, C. J. (2007) Environmental Determinants of Child Mortality in Kenya. World Institute for Development. Research Paper No. $2007 / 83$ 
[8]. Rutstein, S.O (2008) Further Evidence of the Effects of Preceding Birth Intervals on Neonatal, Infant, and Under-Five-Years Mortality and Nutritional Status in Developing Countries: Evidence from the Demographic and Health Surveys. Demographic and Health Research 2008 NO.21

[9]. Singh, R., Bener, A., Singh, K., Dwivedi, S.N. (2008) Child Survival Model in Reference to First Birth Order: Statistical Validation and Prediction. Curr Paediatric Res 2012:16(1):19-25

[10]. UNICEF, (2005) State of the World's Children, New York

[11]. WHO, (2005) The World Health Report 2005. Geneva, World Health Organization

[12]. Worku, Z. (2009) Factors That Affect Under-Five Mortality among South African Children: Analysis of the South African Demographic and Health Survey Data Set . World Congress on Engineering and Computer Science 2009 Vol II WCECS 2009, October 20-22, 2009, San Francisco, USA

[13]. Zimbabwe Central Statistical Office and Macro International Inc, (2007)VZimbabwe Demographic and Health Survey Country Report. Harare: Central Statistical Office.

[14]. Zimbabwe National Statistics Agency, (2009) Multiple Indicator Monitoring Survey (MIMS) 